the detectors were locked on, and the signal gradually decayed over the next $150 \mathrm{~s}$ or so. Near the end of the flight the detectors were displaced about $10^{\circ}$ to take a background reading. A significant drop in count rate was immediately recorded. Seward and his colleagues suggest that this was due to a diffuse source associated with the unocculted part of the nebula. At a temperature $\mathrm{KT} \sim 0.8 \mathrm{keV}$ and luminosity $\sim 10^{36} \mathrm{erg} \mathrm{s}^{-1}$, this puts at least part of the $\mathrm{Crab}$ in line with other X-ray supernova remnants.

Very long baseline interferometry radio observations of the nucleus of NGC1275 (3C84) by E. Preuss (Bonn) and his colleagues contrasted well with the maps shown by A. G. Willis (Leiden) of the large radio galaxies observed from Westerbork. The VLBI observations could be interpreted in terms of a stable map showing $10 \mathrm{com}$ ponents on the scale of $\sim 1$ milliarc second! (This is about the angle subtended by a man on the moon.) At the other extreme, the large scale emission regions of 3 C236 and DA240 could accommodate many Cygnus-As. A compact, highly polarised, and unresolved source has been found at the leading edge of the eastern component of 3C236 which, if not a background source, will be of interest in discriminating between the many theories of radio galaxies.

Many more new and unpublished results and ideas were presented at this very successful conference. In his review of the theory of supernova remnants, S. Gull (Cambridge), showed a memorable radio "photograph" of the supernova remnant Cas A "taken" with the $5 \mathrm{~km}$ telescope. The turbulent detail in the shell of this remnant is as dramatic as any optical picture of the Orion nebula. Popular books will never look the same when these new pictures start replacing the old favourites.

The meeting showed that European astronomy is in a healthy state and it is to be hoped that the European Conference on Astronomy may become an annual event.

\section{$X$ rays and ion transport in liquids}

\section{from Andrew Holmes-Siedle.}

THE use of photographic film to record $\mathrm{X}$ rays has always had the disadvantage that one has to wait to see the image. Another less obvious disadvantage is the "chemical fogging" which impedes perception of fine detail. Direct viewing of fluorescent screens demands high doses to the subject or the use of intensifying systems which, though costly, do not always give enough detail and do not yield a record of the image in "hard copy". There is now growing interest in a method which seems to combine the virtues of each system with cheapness and simplicity and involves some interesting physics. At least two groups are working on it, using the names 'electron radiography' in the USA and 'ionography' in the UK.

The X-ray shadowgraph image is converted into a charge image on a dielectric by the ionisation of a gas or liquid in a chamber and the transport of the positive ions in a straight line to one wall of that chamber, where they collect as stored charge on a dielectric sheet. This charge image can then be developed with a powder in the same way as in the well-known xerographic processes. The transport of the ions is effected by a fairly high applied electric field and no pre-charging of the sheet is necessary. Such an idea may sound simple but it is only recently that a method yielding a satisfactory resolution has been developed.

Early attempts to use electrostatic image formation all involved the use of pre-charged insulator surfaces. Even before the use of film was established, within months of the discovery of $\mathrm{X}$ rays, Righi (The Electrician, 37, 75; 1896) had found that $X$ rays would discharge ebonite and that the pattern could be made visible with powders. The discharging of a selenium plate by $X$ rays was embodied in the original Xerox patents but, despite some investigation in the 1950s, "xeroradiography" has not been widely used. Criscuolo (Non-Destructive Testing, 14, 28-36; 1956) then found that the X-rayinduced conductivity of air over a charged insulator surface could be used to produce an image but that resolution was still poor, mainly because of the small fraction of photons colliding with gas near the plate. The step to a good image was made by increasing both the atomic number and density of atoms above the insulator and by replacing the field from the charged insulator by an applied field, then simply inserting an uncharged insulator foil between the field electrodes and allowing it to intercept and trap the charged species which drifted towards it. Two groups realised the efficiency of this method at about the same time. H. E. Johns, J. W. Boag and others reported favourable results in $1974(B r . J$. Radiol., 47, 519-529) while A. P. Proudian and others presented a verbal description in 1972 and published results in 1974 (Radiology, 110, 667-671).

In both of these publications, the density of atoms near the insulator was increased by using a gas at pressure. Xenon at ten atmospheres proved ideal, since this element has an absorption peak which nicely matches the white radiation spectrum emitted from a 100 $\mathrm{keV} \mathrm{X}$-ray tube. The polarity of field is normally such that the xenon ions bombard and charge the surface of the insulator foil. While the electrons will also produce a charge image, the British workers showed that scattering on the way to the foil caused serious loss of resolution. This team thus used the name ionography for the technique, whilc the American team preferred to call it electron radiography to avoid the historical associations of the former territ.

The American team, Allan et al., have now published (J. appl. Phys., 46, 2766-2767; 1975) details of an improved process, termed "liquid-absorber electron racliography", which avoids the use of gas at high pressure. The extra simplicity of using a liquid transporter medium clearly confers cngineering advantages but physicists should also be intrigued by this new requirement for efficient transport of charge is a condensed phase. The composition of the liquid will have to be tailored to meet several unusual demands. Not only must it be a good X-ray absorber but its nature must be such as to minimise recombination and scattering of the ions as they drift to the foil. Of course, in order to reduce lateral charge movement and "fog" from leakage between the field plates, the liquid must also be an excellent insulator when not being irradiated. Since it is desirable to sweep out the ions rapidly, fields near to those producing avalanche effects in the medium will be favoured. Since the science of charge transport in normally nonconducting materials in the condensed phase is still in its infancy, this new demand for know-how may give a welcome increase of intcrest in the basic processes of transport.

The prediction of the British workers, that a very thin pencil of $X$ rays will produce a spot image of radius about $0.175 \mathrm{~mm}$ at half maximum in highpressure xenon is nicely borne out in thcir experiments, which indicate an image resolution of about 10 line pairs per $\mathrm{mm}$. The results quoted by the US workers for the liquid medium show a stronf: improvement on this result. the resolution for the latter medium being; much better than 10 line pairs per mm. Clearly, charge image radiography has come of age but should still provide some interesting physical problems during its further maturation.

\section{Erratum}

In the article 'Jupiter XIII' (Nature, 257,$178 ; 1975$ ) para 2 , line 20 should read "magnitude about +22 " not "matgnitude about +0.22 ". 